\title{
Study on worn topography of bullet surface based on multi-fractal theory
}

\author{
Bingcheng Wang ${ }^{1,}$, Chang Jing ${ }^{2, b}$ and Litian $\mathrm{Li}^{3, \mathrm{c}}$ \\ ${ }^{1}$ Shenzhen University Shenzhen, China \\ ${ }^{2}$ Guangdong Hengzheng Forensic Clinical Medicine Institute Guangzhou, China \\ ${ }^{3}$ Guangdong Hengzheng Forensic Clinical Medicine Institute Guangzhou, China \\ awbc8636@sina.com, ${ }^{b} 328448404 @ q q . c o m,{ }^{c} 1725025919 @ q q . c o m$
}

\begin{abstract}
Keywords: multi-fractal; fractal dimension; dimension spectrum; bullet surface worn
Abstract. The surface worn date of the bullets shot from three type guns were collected by applying collection instrument, and the worn topography of bullet surface was studied by application of fractal theory, its surface worn show the fractal characteristics. The fractal dimensions about the profile curve of bullet worn surface are calculated with multi-fractal method and the total dimension is obtained by the sum method. On the basis of this, the characteristic parameters are extracted from the multi fractal dimension spectrum and its sum value is obtained. The result shows that worn degree of bullet surface is not same, and its total dimensions and the sum value of the characteristic parameters also are not same, when bullets are shot with different types of gun. Therefore, the total value of the multi-fractal dimensions and the sum value of the characteristic parameters may be used as the index to describe the characteristic of bullet surface worn. This may help to analyze the change characteristics of worn surface topography of bullet, and provide reference for judgment of the gun that shoots such bullet. It provided an identification method for quantitative examination of bullet mark.
\end{abstract}

\section{Introduction}

When the bullets move in the barrel, on the one hand, the pressure from the inside of gun barrel caused plastic deformation; on the other hand ,the bullets and the inner wall of the barrel contact with each other, so they have a relative motion and a frictional wear is generated [1]. The geometric figure reflected by the bullet surface worn is so complex and irregular that it's difficult to describe it accurately. In the past, we make the research on the characteristic of bullet surface worn by using some parameters related to measuring instrument resolution or gage length and measure area, such as arithmetical mean deviation of the profile. These parameters have a positive effect on the understanding of friction and wear, but there are also some shortcomings. In the examination and identification of judicial evidence, the inspection of bullet surface worn is usually by visual comparison. That is, the identification and judgment of the worn shape of bullet recovered from the crime scene or the suspected guns with eyes are not quantitative examinations.

Surface topography was studied by using fractal theory and the research has obtained certain achievement [2.3.4]. In this paper, fractal geometry theory and the literature on the application of fractal research [5] are applied to study the characteristics of the surface topography of bullet worn. The purpose of the study is to apply the fractal theory to the inspection of bullet marks, so that the modern theory and application technology can be combined to deepen the theory of trace inspection. Its practical value is the introduction of new examination techniques for the inspection of the bullet mark in the process of investigating the guns case.

\section{Experimental conditions and data acquisition}

In experimental study, the fired bullets were sampled which are two kinds of bullets fired by three types of guns (the number of 1, 2, 3 gun) with moderate worn, the unit is $\mu m$. Some mark samples of the surface worn were made, and the samples were observed under the stereo microscope. Those 
samples were chosen which can reflect the stability characteristics of the worn marks to undergo digitalized gathering.

Using optical 3D measurement device G4g made in Austria, the mark of the surface worn is digitally collected, and a three-dimensional of wear mark is stored on the computer. In this experiment, the eyepiece magnification is 10 times and the sampling resolution is $1.1 \mu \mathrm{m}$. The stable part of the mark feature is labeled by the application software to get the profile curve which is perpendicular to the mark surface. Data were collected; the collected data was input to the computer to print out profile curve to perpendicular to the wear surface. As is shown in Figure.1.The abscissa is the sampling points of the curve, the ordinate is the profile height value $\mathrm{Z}$ (x).

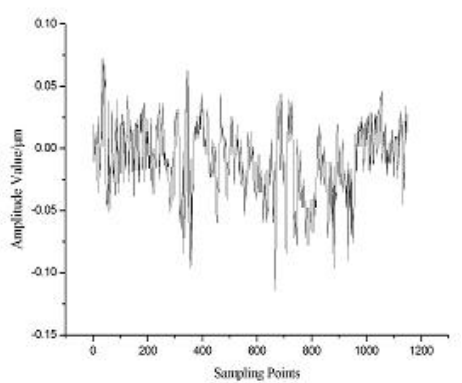

(a)

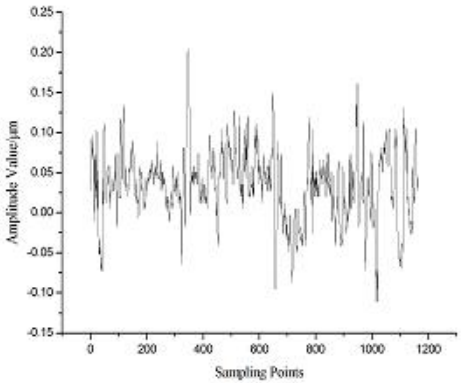

(b)

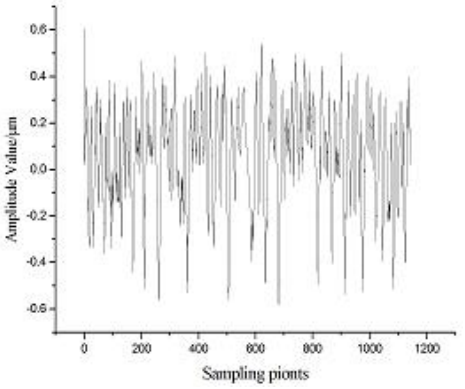

(c)

Figure. 1 Three kinds of profile curve

\section{Multi-fractal theory of contour curve and fractal dimension calculation}

Fractal theory provides the theoretical basis for the description of nonlinear behavior of the system, and it is used to quantitatively characterize the singularity of chaotic attractor. To a certain extent, the fractal dimension make up for the deficiency of the traditional analysis model. In order to describe the nonlinear system behavior characteristics by fractal dimension, the various definitions and calculations methods are put forward, such as the yard stick method, the box dimension method, the power spectrum method, the correlation dimension method, the information dimension method, and the general dimension method, and so on[6]. In this paper, the fractal dimension of the contour curves perpendicular to the bullet surface worn are calculated by the multi-fractal method.

The multi-fractal dimension, the general definition of the fractal dimension, its expression is:

$$
D_{q}=-\lim _{\varepsilon \rightarrow 0} \frac{I_{q}(\varepsilon)}{\ln \varepsilon}
$$

A series of information entropy $I_{q}(\Varangle$ can be calculated according to the formula, a scale-free region is found in $\ln \varepsilon \sim \ln \left[I_{q}(\Varangle]\right.$ Figure, fitting the slope of the segment using the least squares method, the absolute value of this slope is $D_{q}$ of a given value $q$.

According to the formula of the multi-fractal dimension, the dimension spectrum values of three kinds of profile curve is obtained, its dimension spectrum curve is shown in Figure .2:

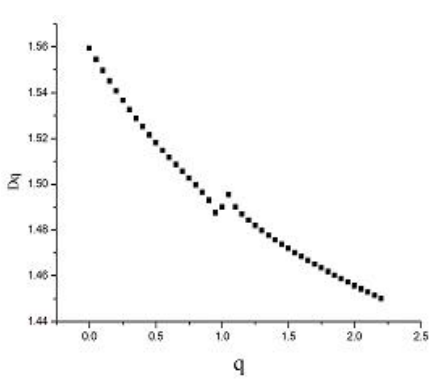

(a)

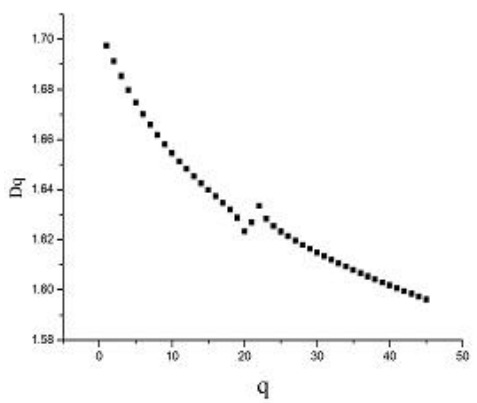

(b)

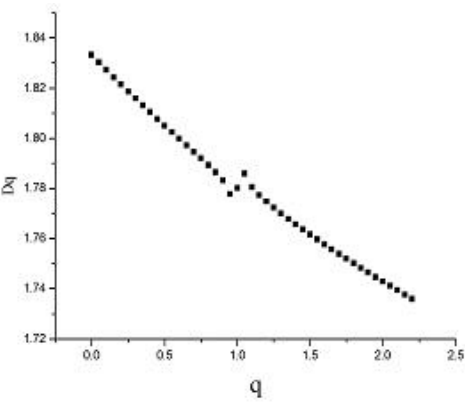

(c)

Figure.2 Three kinds of dimension curve 


\section{Analysis of the profile curve characteristics based on multi-fractal dimension spectrum}

From the multi-fractal dimension spectrum value, characteristic values is extracted such as $D_{0}, D_{0.95}, D_{1}$, $D_{1.05}, D_{2}$, they are as shown in Table 1:

Table 1 characteristic value of the multi-fractal dimension spectrum

\begin{tabular}{llcc}
$D_{q}$ & Sample a & Sample b & Sample c \\
\hline$D_{0}$ & 1.5594 & 1.6974 & 1.8333 \\
$D_{0.95}$ & 1.4875 & 1.6234 & 1.7778 \\
$D_{1}$ & 1.4901 & 1.6269 & 1.7802 \\
$D_{1.05}$ & 1.4955 & 1.6336 & 1.7860 \\
$D_{2}$ & 1.4501 & 1.5962 & 1.7360 \\
\hline
\end{tabular}

In the calculation of the multi-fractal dimension, taking the iteration order of $q=0.05$, a series of fractal dimension is as shown in figure 2, it can be seen from the fractal dimensions shown in the figure, with the continuous increase in the value of q, dimensional numerical showed monotonically decreasing, but the fractal dimension value in the $\mathrm{q}=0.95$ and $\mathrm{q}=1.05$ generated mutations. So the dimension at the two points, and fractal dimension of $D_{0}, D_{1}$ and $D_{2}$ combine together to describe the fault signal feature, the fractal dimension group of identification rules is established. Even if the fractal dimension $\left(D_{0}, D_{0.95}, D_{1}, D_{1.05}\right.$ and $\left.D_{2}\right)$ at q=0, 0.95, 1, 1.05, 2 five special points are taken as a group to distinguish the different vibration state, It can be seen from the results, three kinds of profile curve is not the same, dimension spectra is also different.

In order to describe the characteristics of different profile curves by using the fractal dimension spectrum, the sum of the values of the fractal dimension is expressed as:

$$
D=\sum_{i=1}^{n} \alpha_{i} D_{i}
$$

$\alpha_{i}$ is weight coefficient, in this calculation, $\alpha_{i}=1$.

The multi-fractal dimension spectrum of three kinds of profile curve can be calculated by the formula (2), the results are shown in Table 2:

Table 2 the total value of the multi-fractal dimensions of three kinds of profile curve

\begin{tabular}{lccc}
\hline profile curve & Sample a & Sample b & Sample c \\
\cline { 1 - 1 } $\begin{array}{l}\text { the total value of the } \\
\text { multi-fractal } \\
\text { dimensions }\end{array}$ & 67.1774 & 73.4172 & 80.0984 \\
\hline
\end{tabular}

For table 2 the sum value of the characteristic parameters in the multi fractal dimension spectrum is expressed as:

$$
D_{t}=\sum \beta_{j} D_{j}
$$

$\beta_{i}$ is weight coefficient, in this calculation, $\beta_{i}=1$

the sum values of the characteristic parameters of three kinds of profile curve can be calculated by the formula (3), the results are shown in Table 3. 
Table 3 the sum values of the characteristic parameters of three kinds of profile curve

\begin{tabular}{llcc}
\hline profile curve & Sample a & Sample b & Sample c \\
\hline $\begin{array}{l}\text { the sum value of the } \\
\text { characteristic } \\
\text { parameters }\end{array}$ & 7.4826 & 8.1775 & 8.9133 \\
\hline
\end{tabular}

It can be seen from the results, the total value of the multi-fractal dimensions and the sum value of the characteristic parameters of three kinds of profiles curve are significantly different, and the distinction is large.

\section{Conclusions}

The multi-fractal dimension spectrum values of three kinds of profile curve were calculated. Profile curve is different, dimension spectrum is also different. Dimension value of the spectrum is analyzed when $\mathrm{q}$ values are different. According to the characteristics of the spectrum, the profile curve characteristics are described by characteristic value of five dimensions $\left(D_{0}, D_{0.95}, D_{1}, D_{1.05}\right.$ and $\left.D_{2}\right)$. By using fractal dimension group to identify the profile curve, the recognition rate is more reliable than using a single dimension.

Based on this calculation, the total value of the multi-fractal dimensions and the sum value of the characteristic parameters can be calculated. As the total dimension value is combined with all the dimension information, the sum value of characteristic parameters is combined with 5 characteristic parameters, the profile curve characteristics can be reflected more comprehensively and accurately. These greatly improve the reliability of the recognition of the contour curves formed by different tools. Studies have shown that the total value of the multi-fractal dimensions and the sum value of the characteristic parameters of three kinds of profile curve are significantly different, and the distinction is large. Therefore, they can be used as the characteristic parameters of different profile curve and a criterion for identifying bullets from different guns. This study provides a basis for the inference of shooting Gun, at the same time, also provides a new method for the quantitative examination of the bullet surface worn marks.

\section{Acknowledgements}

This work was financially supported by Nature Science Fund of China (NSFC), No.: 61571307

\section{References}

[1] D. Z .Li, G. A .Li. Technical inspection of bullet marks. (Beijing: Police Education Press 1995)

[2] C Jing B C Wang. The Classification Recognition of Projectiles Wear Mark Based on Support Vector Machine Method. Recent Advances in Computer Science and Information Engineering vol. 1, pp: 389-394, 2012.

[3] L Mou, M Yang. Research on Criminal Tool Wear based on Multi-scale Fractal Feature of the Texture Image. ICCASM 2010,pp:685-688, ISBN: 978-1-4244-7236-9,2010

[4] Y.W Su, W Chen, A B Zhu,et al. Contact and. Wear Simulation Fractal Surfaces, Joural of Xi An Jiaotong University, Vol.47, No7, P52-55, 2014 (In Chinese)

[5] Y X Li, M Z Gao, K Ma. Developments In Calculation Theory of Fractal Dimension of Rough Surface, Advances in Mathematics, Vol.41, No4, P397-408, 2014 (In Chinese)

[6] B. C. Wang, Z Y Wang, C. Jing. Study on Examination Method of Striation Marks Based on Fractal Theory. Applied Mechanics and Materials, 2015, Vol.740: 553-556 